Artigo Original

Original Article

Ruth Magalhães ${ }^{1}$

Maria Cecilia Martinelli lório²

Descritores

Idoso

Deficiência auditiva

Auxiliares de audição

Questionários

Cognição

Keywords

Elderly

Deafness

Hearing aids

Questionnaires

Cognition

Endereço para correspondência:

Ruth Magalhães

Av. Adolfo Pinheiro 760/222A, Santo Amaro, São Paulo (SP), Brasil, CEP: 04734-001.

E-mail: 1ces@terra.com.br

Recebido em: 9/11/2010

Aceito em: 22/2/2011

\section{Avaliação da restrição de participação e de processos cognitivos em idosos antes e após intervenção fonoaudiológica}

\author{
Evaluation of participation restriction and cognitive \\ processes in the elderly before and after the audiologic \\ rehabilitation
}

\section{RESUMO}

Objetivo: Avaliar a restrição de participação em atividades de vida diária e de processos cognitivos em idosos, de acordo com gênero e faixa etária, antes e após intervenção fonoaudiológica. Métodos: Participaram 50 idosos, 23 do gênero feminino e 27 do gênero masculino. Os idosos foram distribuídos em dois grupos etários: Grupo 1 - 24 idosos, com idade entre 60 e 74 anos (11 do gênero feminino e 13 do gênero masculino); Grupo 2 - 26 idosos com idade igual ou superior a 75 anos (12 do gênero feminino e 14 do gênero masculino). Os idosos foram avaliados antes após um ano de adaptação da prótese auditiva, por meio do questionário HHIE e o teste MEEM. Foram realizados acompanhamentos bimestrais totalizando sete reuniões, a fim de garantir a utilização efetiva da prótese. Quanto à estatística, foram realizadas análises descritivas e análise de variância. Resultados: Houve redução da restrição de participação nas escalas emocional e social do HHIE no período pós-intervenção, nos dois grupos, em indivíduos de ambos os gêneros. Os resultados do teste MEEM foram melhores no período pós-intervenção, independentemente do gênero e idade do participante. Conclusão: Há menor autopercepção das restrições de participação em atividades de vida diária após intervenção fonoaudiológica. Além disso, idosos de diferentes idades e de ambos os gêneros referem diminuição de restrições cognitivas após adaptação de prótese auditiva.

\begin{abstract}
Objective: To evaluate the restriction of participation in activities of daily living and cognitive processes in older adults, according to gender and age, before and after rehabilitation. Methods: The study included 50 individuals, 23 females and 27 males. The elderly were divided into two groups: Group 1 to 24 individuals aged between 60 and 74 years (11 females and 13 males), Group 2 - 26 elderly aged above 75 years (12 of female and 14 male). The elderly were evaluated before one year after the adaptation of hearing aids through the HHIE test and MMSE. Bimonthly follow-ups were conducted a total of seven meetings, to ensure the effective use of the prosthesis. As for the statistical analysis were performed descriptive and statistical analysis. Results: There was a reduction of the restriction of participation in social and emotional scales HHIE post-intervention in both groups, individuals of both genders. The MMSE test results were better in the post-intervention, regardless of gender and age of the participant. Conclusion: There is little perception of the restrictions on participation in activities of daily living after rehabilitation. Moreover, the elderly of different ages and both genders decreased cognitive constraints refer after hearing aid fitting.
\end{abstract}

Trabalho realizado na Universidade Federal de São Paulo - UNIFESP - São Paulo (SP), Brasil.

(1) Universidade Federal de São Paulo - UNIFESP - São Paulo (SP), Brasil.

(2) Departamento de Fonoaudiologia, Universidade Federal de São Paulo - UNIFESP - São Paulo (SP), Brasil. 


\section{INTRODUÇÃO}

A deficiência auditiva é uma das principais alterações sensoriais que acometem o indivíduo durante o processo de envelhecimento. A associação do envelhecimento com a deficiência auditiva causa mudanças físicas, psicológicas e sociais, comprometendo a comunicação e a interação do idoso com a sociedade.

A presbiacusia, que é o envelhecimento do sistema auditivo, promove diminuição na habilidade de compreender, redução na inteligibilidade de fala e comprometimento do processo de comunicação verbal, reduzindo gradativamente o contato social do idoso e podendo causar alterações emocionais. Dentre todas as privações sensoriais, a incapacidade de se comunicar com outras pessoas devido à deficiência auditiva, pode ser uma das conseqüências mais frustrantes para o idoso ${ }^{(1)}$.

Autores relatam que o primeiro sinal da presbiacusia é o momento em que o idoso não consegue mais acompanhar uma conversa, principalmente em ambiente com ruído competitivo, como televisão, música ou outras conversas paralelas. Estas restrições repercutem de forma negativa no relacionamento com outras pessoas e podem gerar um quadro de depressão e isolamento comprometendo a qualidade de vida do idoso ${ }^{(2)}$.

As alterações de saúde ocasionadas pelo envelhecimento podem afetar a autonomia e independência do idoso e, consequentemente, causar prejuízos no convívio social e no bem estar. Sendo assim, a perda auditiva pode restringir as atividades de vida diária do idoso, aumentando assim o declínio funcional. Além disso, estudos demonstraram que o grau da perda auditiva pode afetar o desempenho cognitivo do paciente idoso ${ }^{(3,4)}$.

A adaptação de próteses auditivas visando melhora do desempenho cognitivo vem sendo estudada. Atualmente, pesquisas revelam que o uso de próteses auditivas proporciona melhora da atenção, reversão da condição de isolamento e da dificuldade de comunicação e melhora na qualidade de vida ${ }^{(5-7)}$.

Assim, torna-se importante estudar a relação entre a deficiência auditiva e o desempenho cognitivo do paciente idoso. Com base nestas considerações, o objetivo desta pesquisa foi avaliar a restrição de participação em atividades de vida diária e de processos cognitivos em idosos, de acordo com gênero e faixa etária, antes e após intervenção fonoaudiológica

\section{MÉTODOS}

Este estudo foi avaliado e aprovado pelo Comitê de Ética em Pesquisa da Universidade Federal de São Paulo (UNIFESP), sob o número 0913/08. A pesquisa foi realizada na Associação de Prevenção, Atendimento Especializado e Inclusão da Pessoa com Deficiência (APRAESPI) na cidade de Ribeirão Pires - SP. Os pacientes foram orientados e assinaram o Termo de Consentimento Livre e Esclarecido elaborado com base na resolução 196/96 da CONEP (Comissão Nacional de Ética em Pesquisa).

Os critérios de elegibilidade para composição da amostra foram: possuir idade igual ou superior a 60 anos, apresentar perda auditiva neurossensorial bilateral simétrica de grau a severo e Índice Percentual de Reconhecimento de Fala (IPRF) superior a 50\%, ter indicação de adaptação de próteses auditivas binaural, não apresentar evidências de outros comprometimen- tos e ser alfabetizado.

A amostra constou de 50 idosos, sendo 23 do gênero feminino (46\%) e 27 (54\%) do gênero masculino. Vinte e quatro (48\%) tinham idade na faixa de 60 a 74 anos e $26(52 \%)$ na faixa de 75 anos ou mais. Considerando as três categorias de escolaridade observou-se que nenhum dos idosos da amostra era analfabeto, 49 (98\%) tinham escolaridade de um a 11 anos e apenas um (2\%) de 12 ou mais anos de escolaridade.

Os idosos foram subdivididos em dois grupos etários: Grupo 1 - 24 idosos (48\%), com idade entre 60 e 74 anos (11 do gênero feminino e 13 do gênero masculino); Grupo 2 - 26 idosos (52\%), com idade igual ou superior a 75 anos (12 do gênero feminino e 14 do gênero masculino). Foi aplicado o questionário HHIE (Hearing Handicap Inventory for the Elderly $)^{(8)}$, que foi desenvolvido para avaliar as conseqüências psicossociais, emocionais e sociais decorrentes da perda auditiva no paciente idoso. O questionário HHIE foi originalmente escrito na Língua Inglesa e adaptado para o Português ${ }^{(9)}$. Para aplicação do questionário, foram utilizados papel e lápis, já que todos os idosos eram alfabetizados.

O HHIE é constituído por 25 perguntas, dentre as quais, 13 exploram as conseqüências emocionais da deficiência auditiva e 12 os efeitos sociais e situacionais da deficiência. $\mathrm{O}$ grau de classificação das restrições de participação foi determinado a partir do escore global considerando as 25 questões, seguindo a pontuação das respostas, em que se atribui quatro pontos para a resposta "sim", dois pontos para "às vezes" e zero ponto para "não". Somando todos os pontos atribuídos, o escore total pode variar em percentuais de $0 \%$ (sugerindo handicap ausente) a $100 \%$ (sugerindo total percepção do handicap). Quanto maior o escore, maior é a percepção do indivíduo em relação a sua restrição de participação, ou seja, maiores são as dificuldades auditivas e não auditivas impostas pela deficiência. As pontuações são distribuídas da seguinte forma de acordo com a percepção da restrição: não há percepção (zero a 16), percepção leve-moderada (18 a 42) e percepção severo-significativa (>42).

Foi aplicado também o Teste Mini Exame do Estado Mental (MEEM), elaborado com o objetivo de avaliar processos cognitivos. O teste foi traduzido e adaptado para o Português ${ }^{(10,11)}$. O teste Mini Exame do Estado Mental contém questões relativas à memória imediata, memória de evocação, orientação temporal, orientação espacial, atenção e cálculo e linguagem. Este teste fornece informações sobre os processos cognitivos, contendo questões agrupadas em sete categorias, que avaliam funções cognitivas específicas como orientação temporal (cinco pontos), orientação espacial (cinco pontos), registro de palavras (três pontos), atenção e cálculo (cinco pontos), recordação das palavras (três pontos), linguagem (oito pontos) e capacidade construtiva visual (um ponto). O escore do MEEM pode variar de zero, que indica o maior grau de comprometimento cognitivo dos pacientes, a 30 pontos, que corresponde à maior capacidade cognitiva. Devido à necessidade de utilização de pontos de corte diferenciados, segundo a escolaridade de cada indivíduo, foram consideradas como alteradas as seguintes situações: analfabetos com pontuação inferior a 13 pontos; indivíduos com escolaridade média (até oito anos de instrução formal) pontuação inferior a 18 pontos; indivíduos com alta escolaridade (mais de oito anos de 
instrução) pontuação inferior a 26 pontos $^{(12)}$. O teste foi aplicado com a orientação da pesquisadora, a fim de que fosse garantida a compreensão dos itens propostos.

Foram agendadas reuniões periódicas bimestrais, totalizando sete reuniões, com o objetivo de acompanhar o processo de adaptação das próteses auditivas. Nestas reuniões, os pacientes receberam orientações sobre uso, cuidados e manuseio com as próteses e orientações quanto às estratégias de comunicação. No momento da primeira reunião os idosos receberam por escrito todas as informações sobre o objetivo da pesquisa e um cartão contendo todas as datas das reuniões agendadas até o final da pesquisa. Também na primeira reunião, os idosos responderam o questionário HHIE e o teste MEEM. A partir da segunda reunião os idosos já estavam adaptados com suas próteses auditivas. Durante as outras reuniões os idosos esclareceram dúvidas quanto ao uso, manuseio e cuidados, utilização de acessórios com desumidificador, bombinha de ar para limpeza do molde, uso do telefone e estratégias de comunicação. $\mathrm{Na}$ última reunião, após aproximadamente um ano de utilização da prótese, todos os idosos responderam novamente o questionário HHIE e o teste MEEM.

\section{Método estatístico}

Foram construídas tabelas com valores de estatísticas descritivas para os escores do questionário HHIE e para o teste MEEM por período, gênero e faixa etária. Para comparar as médias dos escores nos dois períodos de avaliação, gênero e faixa etária, foi aplicada a técnica de Análise de Variância com medidas repetidas ${ }^{(13)}$. As médias das diferenças nos períodos pré e pós foram estimados e construídos intervalos de confiança de $95 \%$. O teste MEEM foi também categorizado em Normal e Alterado, considerando os critérios estabelecidos ${ }^{(12)}$, de acordo com a pontuação e escolaridade. As probabilidades de ocorrência dessas categorias no pré e pós foram comparadas por meio do teste de $\mathrm{McNemar}{ }^{(14)}$. Em cada teste de hipótese foi adotado o nível de significância de 0,05 e os valores de p significativos foram destacados com o símbolo asterisco (*).

\section{RESULTADOS}

Foram estabelecidas as estatísticas descritivas para os escores total do questionário HHIE antes e após intervenção fonoaudiológica. $\mathrm{O}$ escore médio obtidos pelos idosos no período pré-intervenção $(32,9 \%)$ foi menor do que no período pós $(8,8 \%)$ (Tabela 1$)$.

Tabela 1. Estatística descritiva para a pontuação total do HHIE nos períodos pré e pós-intervenção fonoaudiológica

\begin{tabular}{lcccccc}
\hline Variável & $\mathrm{n}$ & Média & $\mathrm{DP}$ & Mínimo & Mediana & Máximo \\
\hline Total pré & 50 & 32,9 & 5,9 & 20 & 34 & 42 \\
Total pós & 50 & 8,8 & 5,1 & 0 & 8 & 22 \\
\hline
\end{tabular}

Pré $x$ Pós $-p<0,0001^{*}$ (Análise de variância com medidas repetidas) Legenda: $\mathrm{DP}=$ desvio-padrão

Após, foram estabelecidas as estatísticas descritivas para os escores do questionário HHIE separadamente, inicialmente relativos à Escala Emocional, antes e após a intervenção para os dois grupos etários e gênero. Aplicou-se a Análise de Variância para investigar se houve diferença entre os escores obtidos nos dois grupos etários, gêneros e condições pré e pós-intervenção. A diferença entre os escores médios relativos à Escala Emocional do HHIE nos períodos pré e pós intervenção, dependeu do gênero $(\mathrm{p}=0,021)$ e da faixa etária $(\mathrm{p}=0,008)$ (Tabela 2).

Tabela 2. Desempenho na escala Emocional do HHIE de acordo com gênero e faixa etária, pré e pós intervenção fonoaudiológica

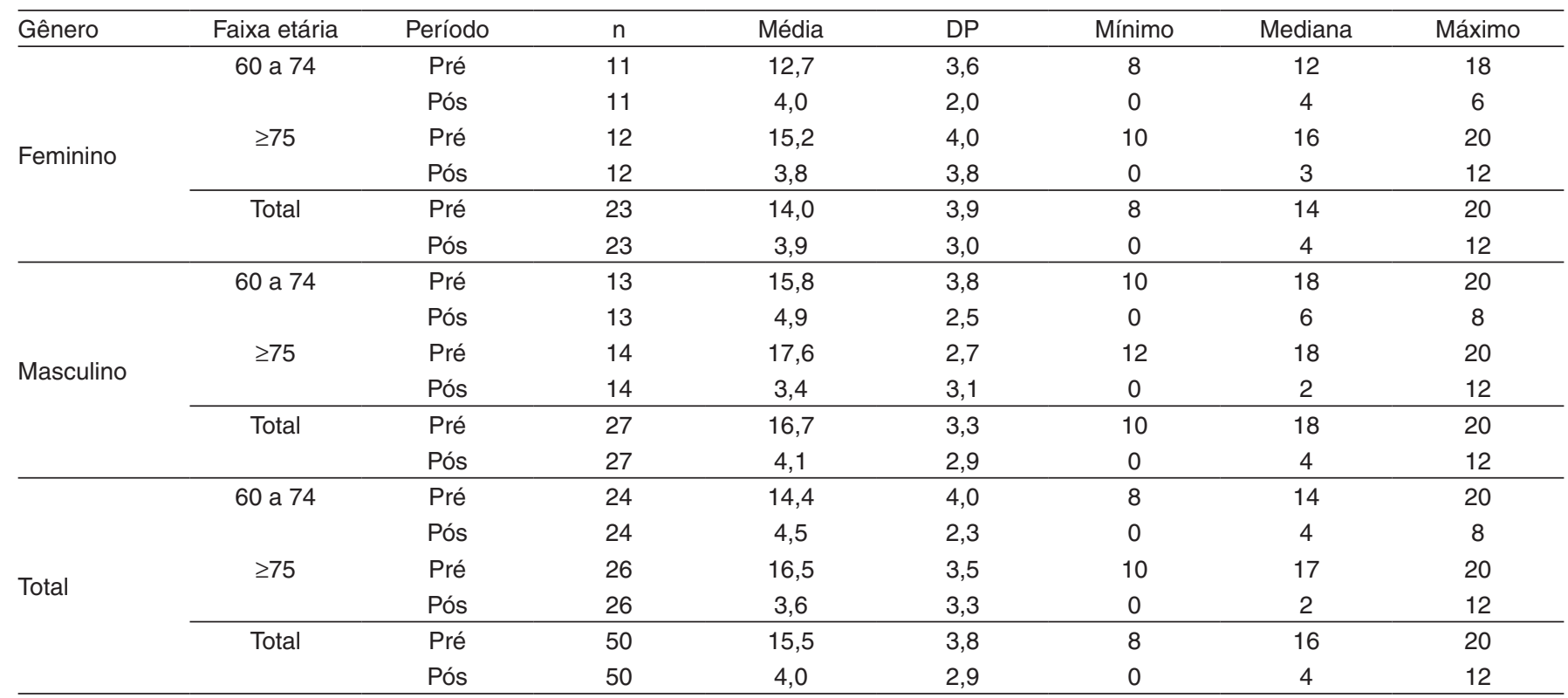

Análise de variância $(p \leq 0,05)$ : Pré $x$ Pós $x$ Gênero $\rightarrow p=0,021^{*}$. Gênero feminino $x$ Pré $x$ Pós $\rightarrow p<0.0001^{*}$. Gênero masculino $x$ Pré $x$ Pós $\rightarrow p<0.0001^{\star}$. Pré $x$ Gênero feminino $x$ Gênero masculino $\rightarrow p=0,003^{*}$. pós $x$ Gênero feminino $x$ Gênero masculino $\rightarrow p>0,999$. Pré $x$ Pós $x$ Faixa etária $\rightarrow p=0,008^{*}$. $\mathrm{G} 1$ (60 a 74 anos) $x$ Pré $x$ Pós $\rightarrow p<0.0001^{*}$. G2 (75 anos ou + ) x Pré x Pós $\rightarrow p<0.0001^{*}$. Pré x G1 (60 a 74 anos) x G2 $\left(\geq 75\right.$ anos) $\rightarrow p=0,026^{\star}$. Pós x G1 (60 a 74 anos) x G2 ( $\geq 75$ anos) $\rightarrow p=0,916$

Legenda: DP = desvio-padrão 
O estudo da diferença entre os escores médios relativos à Escala Social/Situacional nos períodos pré e pós-intervenção dependeu do gênero $(\mathrm{p}=0,004)$, e não ocorreram diferenças entre os dois grupos etários $(\mathrm{p}=0,061)$, independentemente do período ( $\mathrm{p}=0,076$ ). No período pré, os escores médios calculados para os idosos do gênero masculino foram maiores do que do gênero feminino $(\mathrm{p}<0.0001)$ (Tabela 3$)$.

$\mathrm{Na}$ segunda etapa da pesquisa, foram estabelecidas as estatísticas descritivas e análise de variância para os escores obtidos no teste Mini Exame do Estado Mental. Os resulta- dos obtidos demonstraram que os escores médios obtidos no período pós-intervenção foram maiores que no período pré $(p<0,001)$, revelando resultado significativo. Esta diferença ocorreu independentemente do gênero $(\mathrm{p}=0,279)$ e faixa etária $(\mathrm{p}=0,997)$ do idoso (Tabela 4).

As distribuições de freqüência e porcentagens conjuntas do MEEM foram feitas de acordo com o resultado, que poderia ser Normal e Alterado. Observou-se que a probabilidade de ocorrência de resultado alterado no período pós-intervenção é menor do que no período pré-intervenção ( $\mathrm{p}<0.0001$ ) (Tabela 5).

Tabela 3. Desempenho na escala Social/Situacional do HHIE de acordo com gênero e faixa etária pré e pós intervenção fonoaudiológica

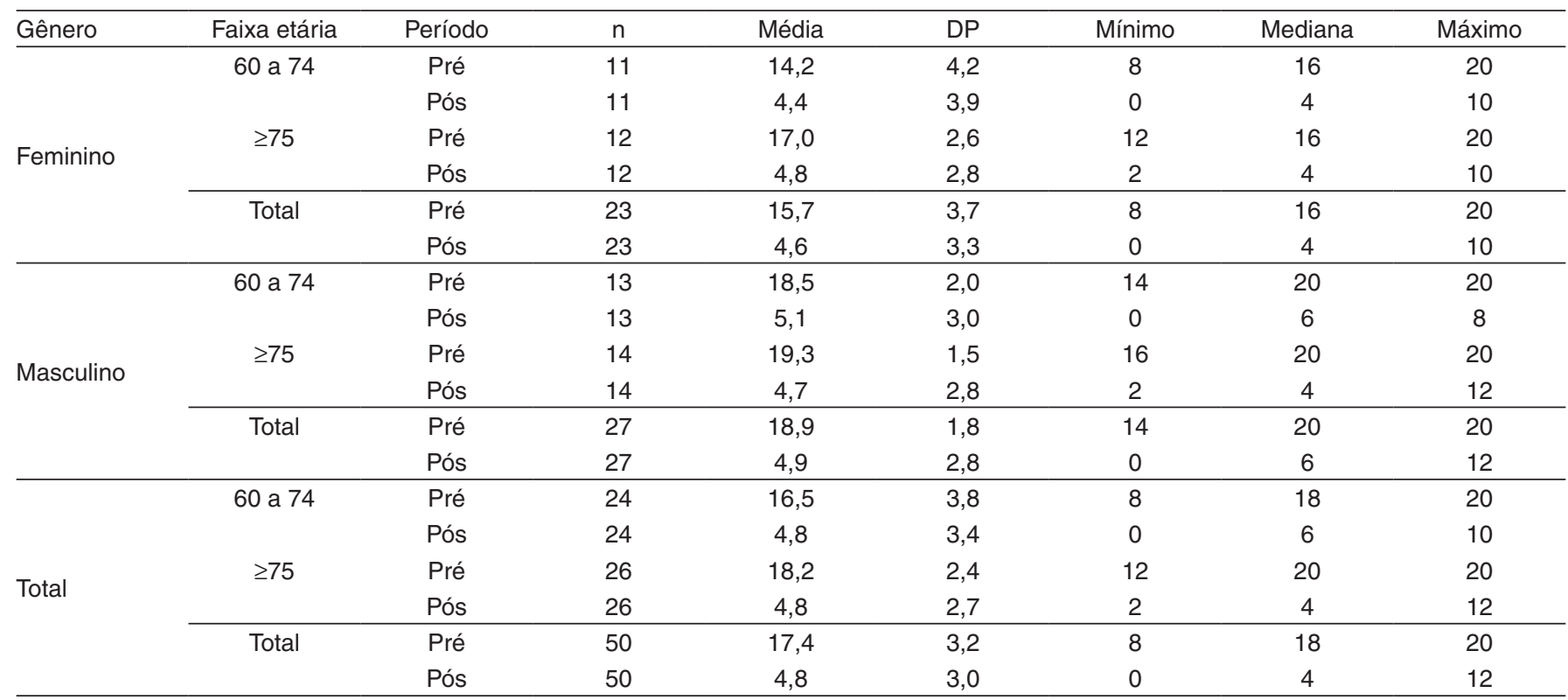

Análise de variância $(p \leq 0,05)$ : Pré $x$ Pós $x$ Gênero $\rightarrow p=0,004^{*}$. Gênero feminino $x$ Pré $x$ Pós $\rightarrow p<0,0001^{*}$. Gênero masculino $x$ Pré $x$ Pós $\rightarrow p<0,0001^{*}$. Pré $x$ Gênero feminino $x$ Gênero masculino $\rightarrow p<0,0001^{*}$. Pós $x$ Gênero feminino $x$ Gênero masculino $\rightarrow p>0,999$. Pré $x$ Pós $x$ Faixa etária $\rightarrow p=0,061$. G1 (60 a 74 anos) x Pré x Pós $\rightarrow p=0,076$. G2 (75 anos ou + ) x Pré $\times$ Pós $\rightarrow p=0,076$

Legenda: $\mathrm{DP}=$ desvio-padrão

Tabela 4. Desempenho no MEEM de acordo com gênero e faixa etária pré e pós-intervenção fonoaudiológica

\begin{tabular}{|c|c|c|c|c|c|c|c|c|}
\hline Gênero & Faixa etária & Período & $\mathrm{n}$ & Média & $\mathrm{DP}$ & Mínimo & Mediana & Máximo \\
\hline \multirow{6}{*}{ Feminino } & 60 a 74 & Pré & 11 & 21,4 & 3,9 & 16 & 20 & 29 \\
\hline & & Pós & 11 & 25,5 & 3,2 & 20 & 26 & 30 \\
\hline & $\geq 75$ & Pré & 12 & 20,4 & 3,8 & 16 & 20,5 & 26 \\
\hline & & Pós & 12 & 24,3 & 3,3 & 20 & 24,5 & 29 \\
\hline & Total & Pré & 23 & 20,9 & 3,8 & 16 & 20 & 29 \\
\hline & & Pós & 23 & 24,9 & 3,2 & 20 & 26 & 30 \\
\hline \multirow{6}{*}{ Masculino } & 60 a 74 & Pré & 13 & 22,7 & 4,3 & 16 & 22 & 28 \\
\hline & & Pós & 13 & 25,9 & 3,6 & 20 & 28 & 30 \\
\hline & $\geq 75$ & Pré & 14 & 21,9 & 3,9 & 17 & 21 & 29 \\
\hline & & Pós & 14 & 25,4 & 3,4 & 17 & 25,5 & 29 \\
\hline & Total & Pré & 27 & 22,3 & 4,0 & 16 & 22 & 29 \\
\hline & & Pós & 27 & 25,7 & 3,4 & 17 & 26 & 30 \\
\hline \multirow{6}{*}{ Total } & 60 a 74 & Pré & 24 & 22,1 & 4,1 & 16 & 21,5 & 29 \\
\hline & & Pós & 24 & 25,8 & 3,3 & 20 & 26 & 30 \\
\hline & $\geq 75$ & Pré & 26 & 21,2 & 3,8 & 16 & 20,5 & 29 \\
\hline & & Pós & 26 & 24,9 & 3,3 & 17 & 25 & 29 \\
\hline & Total & Pré & 50 & 21,6 & 3,9 & 16 & 21 & 29 \\
\hline & & Pós & 50 & 25,3 & 3,3 & 17 & 26 & 30 \\
\hline
\end{tabular}

Análise de variância $(p \leq 0,05)$ : Pré $x$ Pós $\rightarrow p<0,001^{*}$. Pré $x$ Pós $x$ Gênero $\rightarrow p=0,279$. Pré $x$ Pós $x$ Faixa etária $\rightarrow p=0,997$.

Legenda: DP = desvio-padrão 
Tabela 5. Distribuição do número de idosos de acordo com os resultados do MEEM (normal e alterado) nos períodos pré e pós-intervenção fonoaudiológica

\begin{tabular}{lcccc}
\hline \multirow{2}{*}{ Pré } & & \multicolumn{2}{c}{ Pós } & \multirow{2}{*}{ Total } \\
\cline { 3 - 4 } & & Alterado & Normal & \\
\hline Alterado & $\mathrm{n}$ & 7 & 19 & 26 \\
& $\%$ & 14 & 38 & 52 \\
Normal & $\mathrm{n}$ & & 24 & 24 \\
& $\%$ & & 48 & 48 \\
\hline Total & $\mathrm{n}$ & 7 & 43 & 50 \\
& $\%$ & 14 & 86 & 100 \\
\hline
\end{tabular}

Pré x Pós: $\mathrm{p}<0,0001^{*}$ (Teste de McNemar)

\section{DISCUSSÃO}

Inicialmente foram calculadas as estatísticas descritivas para o escore total do HHIE nos períodos pré e pós-intervenção. Os resultados mostraram que no momento pré-intervenção havia uma percepção moderada em relação à restrição de participação em atividades de vida diária. No entanto, no momento pósintervenção não houve percepção desta restrição.

Nos vários estudos que utilizaram o questionário HHIE, os autores relatam que para minimizar as reações psicossociais decorrentes da deficiência auditiva no idoso, tornou-se necessária a inclusão destes idosos em programas de reabilitação auditiva. Após a participação no programa, os idosos apresentam efetiva redução da restrição de participação, fato que demonstra a importância da adaptação de próteses auditivas ${ }^{(15)}$. Os achados da presente pesquisa demonstram que os idosos apresentam significativa redução da autopercepção quanto às restrições de participação após um ano de utilização das próteses auditivas, sendo esta redução independente de gênero e faixa etária. Atribui-se esta melhora simplesmente à adaptação e utilização efetiva das próteses auditivas, aspecto já mencionado em inúmeras pesquisas ${ }^{(15-17)}$. No entanto, é importante destacar que neste estudo houve o acompanhamento bimestral dos pacientes. Este procedimento, sem dúvida alguma, pode ter influenciado nos resultados satisfatórios obtidos.

A análise dos escores relativos à escala Emocional do questionário HHIE revelou percepção da restrição de participação menor no período pós-intervenção. No entanto, no período pré-intervenção, o escore médio obtido por idosos do gênero masculino foi maior que os obtidos por aqueles do gênero feminino, demonstrando que os homens idosos apresentam maior grau de percepção em relação às restrições do que as mulheres idosas. Já a análise da restrição de participação segundo a variável faixa etária revelou escore médio menor no período pós- intervenção. $\mathrm{O}$ decréscimo médio entre os períodos pré e pós-intervenção foi maior em idosos com idade igual ou superior a 75 anos, revelando que estes apresentam maior grau de percepção de suas restrições no período pré e obtêm maiores benefícios após intervenção fonoaudiológica.

A análise dos resultados relativos à escala Social/Situacional revelou que o decréscimo médio ocorrido entre os períodos pré e pós-intervenção foi maior em idosos do gênero masculino.
Tais dados demonstram, novamente, que os homens idosos apresentam maior grau de percepção em relação às restrições de participação e referem benefícios significativos quanto a este aspecto após intervenção fonoaudiológica.

Estudos revelam que idosos do gênero masculino apresentam melhor percepção em relação às restrições do que os idosos do gênero feminino ${ }^{(16)}$, resultados semelhantes aos obtidos da presente pesquisa. Acredita-se que os idosos do gênero masculino apresentaram maior percepção pois, culturalmente, em nossa sociedade, os homens, mesmo idosos, representam o papel do provedor da família, o que pode exacerbar a percepção das restrições de participação.

Estudos mostram que o beneficio da utilização da prótese auditiva pode ser mensurado por meio da avaliação de redução de restrição de participação e pode ser avaliado após seis semanas de utilização efetiva das próteses, período suficiente para demonstrar o beneficio proporcionado ${ }^{(17)}$. Na presente pesquisa, a reavaliação foi feita após quase um ano de adaptação, tempo considerado suficiente para reaplicação do questionário.

Quanto à análise dos resultados do teste MEEM, verificouse que após a intervenção os escores médios foram melhores do que no momento pré-intervenção. Assim, pode-se dizer que o desempenho cognitivo dos idosos, avaliado por este instrumento de triagem, melhorou após intervenção, independentemente de gênero e faixa etária.

Pesquisas demonstram que há relação entre a deficiência auditiva e o desempenho cognitivo dos pacientes idosos e que a reabilitação por meio da adaptação de próteses auditivas contribui para a melhora do desempenho cognitivo. Em estudo realizado anteriormente, apesar de os idosos do gênero feminino apresentarem maiores escores, o que indica melhor desempenho, os resultados não foram significativos ${ }^{(4)}$. Tais achados são semelhantes aos obtidos na presente pesquisa.

O teste MEEM foi analisado, ainda, quanto a classificação dos resultados segundo as categorias normal e alterado ${ }^{(12)}$. Esta análise demonstrou que dos 26 indivíduos (52\%) com resultados alterados no período pré-intervenção fonoaudiológica, 19 idosos (38\%) apresentaram resultado normal no período pósintervenção. Sendo assim, verificou-se que $52 \%$ dos idosos que obtiveram resultados alterados no período pré e somente $14 \%$ apresentaram resultados alterados no período pós.

Pesquisas revelam que, para o processo de comunicação ser bem sucedido, faz-se necessário que o ouvinte acumule ao longo da vida, informação com base na entrada sensorial (bottom-up) e interprete cognitivamente a intenção do falante (top-down). Pesquisadores relatam que ocorre uma grande ativação cerebral quando se torna necessário ouvir em ambientes de difícil escuta. Para compreender a fala em ambientes acusticamente desfavoráveis, o idoso com perda auditiva neurossensorial necessita de um maior esforço, pois utiliza predominantemente os recursos cognitivos para interpretação da informação, o que pode levar ao estresse. Uma deficiência em qualquer entrada sensorial e/ou nos processos cognitivos necessários para interpretação dos sons da fala acarreta obstáculos para a comunicação. Assim, quando a entrada está comprometida, mais recursos cognitivos são necessários para a compreensão da fala, ativando processos compensatórios para 
obter melhor desempenho. Estudos revelam ainda que, quanto maior a idade do paciente idoso e menor a escolaridade, pior é o seu desempenho cognitivo ${ }^{(18,19)}$.

Os resultados encontrados na presente pesquisa evidenciaram que houve melhora no desempenho dos idosos no teste MEEM após um ano de uso das próteses auditivas o que é bastante instigante, já que não se espera melhora da cognição com a utilização das próteses. No entanto, o fato de os idosos estarem mais atentos devido à melhora da sensibilidade auditiva e consequentemente terem menor exigência cognitiva para compreensão da fala, pode ter promovido uma melhora no desempenho cognitivo mensurado pelo teste MEEM.

\section{CONCLUSÃO}

Após a análise crítica dos resultados é possível concluir que, quanto à restrição de participação mensurada pelo HHIE, há menor autopercepção das restrições de participação após a intervenção fonoaudiológica. No entanto, os idosos do gênero masculino apresentam maior autopercepção das restrições de participação no período pré-intervenção fonoaudiológica. Os idosos mais velhos apresentam maior autopercepção das restrições no período pré-intervenção fonoaudiológica. Quanto aos processos cognitivos, idosos apresentam maiores escores, ou seja, melhores resultados nos processos cognitivos após a intervenção fonoaudiológica, independentemente das variáveis gênero e faixa etária.

\section{REFERÊNCIAS}

1. Russo ICP. Distúrbios da audição: a presbiacusia. In: Russo ICP. Intervenção fonoaudiológica na terceira idade. Rio de Janeiro: Revinter; 1999. p. 51-92.

2. Bilton TL. A deficiência auditiva do idoso e sua implicação na comunicação [dissertação]. São Paulo: Pontifícia Universidade Católica de São Paulo; 1989.

3. Anderson MIP, Assis M, Pacheco LC, Silva EAS, Menezes IS, Duarte T, et al. Saúde e qualidade de vida na terceira idade. Textos Envelhecimento. 1998;1(1):23-43.
4. Kopper H, Teixeira AR, Dorneles S, Desempenho cognitivo em um grupo de idosos: influência de audição, idade, sexo e escolaridade. Arq Int Otorrinolaringol. 2009;13(1):39-43.

5. Souza RMM. O impacto da eficiência auditiva na qualidade de vida do idoso, antes e um ano após a adaptação de próteses auditivas [dissertação]. São Paulo: Universidade Federal de São Paulo. Escola Paulista de Medicina; 2010.

6. Allen NH, Burns A, Newton V, Hickson F, Ramsden R, Rogers J, et al. The effects of improving hearing in dementia. Age Ageing. 2003;32(2):189-93.

7. Silva AS, Venites JB, Bilton TL. A relação entre o uso do aparelho de amplificação sonora individual - AASI - e a melhora da função cognitiva no envelhecimento. Distúrb Comun. 2002;14(1):63-89.

8. Ventry IM, Weisnstein B. Identification of elderly people with hearing problems. ASHA. 1983;25(7):37-42.

9. Wieselberg MB. A auto-avaliação do handicap em idosos portadores de deficiência auditiva: o uso do H.H.I.E [dissertação]. São Paulo: Pontifícia Universidade Católica de São Paulo; 1997.

10. Folstein MF, Folstein SE, McHugh PR. "Mini-mental state". A practical method for grading the cognitive state of patients for the clinician. J Psychiatr Res. 1975;12(3):189-98.

11. Bertolucci PHF, Mathias SC, Brucki SMD, Carrilho PEM, Okamoto IH, Nitrini R. Proposta de padronização do Mini-Exame do Estado Mental (MEEM): estudo piloto cooperativo (FMUSP/EPM). Arq Neuropsiquiatr. 1994;52:Supl 1.

12. Bertolucci PHF, Brucki SMD, Campacci SR, Juliano Y. O mini-exame do estado mental em uma população geral: impacto da escolaridade. Arq Neuropsiquiatr. 1994;52(1):1-7.

13. Kutner MH, Neter J, Nachtshein CJ, Li W. Applied linear statistical models. 5th ed. Boston: McGraw Hill; 2005.

14. Fisher LD, Belle VG. Biostatistics. New York: Wiley; c1993.

15. Marques ACO, Kozlowski L, Marques JM. Reabilitação auditiva no idoso. Rev Bras Otorrinolaringol. 2004;70(6):806-11.

16. Pinzan-Faria VM, Iório MCM. Sensibilidade auditiva e autopercepção do handicap: um estudo em idoso. Distúrb Comum. 2004;16(3):289-99.

17. Buzo BC, Ubrig MT, Novaes BC. Adaptação de aparelho de amplificação sonora individual: relações entre a auto-percepção do handicap auditivo e a avaliação da percepção de fala. Distúrb Comum. 2004;16(1):17-25.

18. Schum DJ, Beck DL. Negative synergy: hearing loss and aging. Audiology Online [Internet]. June 23, 2008. [cited 2009 Jul 12]. Available from: http://www.audiologyonline.com/articles/article_detail. asp?article_id=2045

19. Diniz BSO, Volpe FM, Tavares AR. Nível educacional e idade no desempenho no mini exame do estado mental em idosos residentes na comunidade. Rev Psiquiatr Clín (São Paulo). 2007;34(1):13-7. 